\title{
Covid-pandemia: Learning from mistakes during the pandemic?
}

\author{
Andrea Bellone \\ Department of Emergency Medicine, ASST-Grande Ospedale Metropolitano Niguarda, Milano, Italy
}

\begin{abstract}
Hospitals managing the pandemic more successfully were the ones which started working on it ahead of times: so, let's look back at it. Those who acted early, differentiating routes within the Emergency Department (EDpt), creating different areas with varying intensity of care; those who were quick in converting areas of mid-intensive care into Sub-Intensive Care Units (SICU) where to allocate only patients in need of non-invasive ventilation; those who quickly converted internal medicine areas into Covid-19 medicine ones; those who converted SICU into Intensive Care Units (ICU); those who created new ICUs; ultimately, those who adapted hospitals to handle both the extraordinary pressure that hit Emergency Rooms (ERs) and many critical patients (yellow and red codes) still coming in, keeping ERs decongested and therefore able to work safely, guaranteed a fair treatment for all patients and probably improved the prognosis. In reality, as tragically recounted in a May 2020 article on NEJM, ${ }^{1}$ this was not the case. Some hospitals in Lombardy and Piacenza quickly faced major difficulties, despite the heroic efforts of health workers. In sequence: i) The sudden waves of critically ill people accessing the ER required many of them to be admitted to ICUs, quickly exhausting all availabilities before being able to create new ones. ii) A large proportion of patients concentrated in the ER, where they began to be oxygenated and non-invasively ventilated. The medical area of the hospital found it difficult to accommodate patients from the ER, both for an atavistic lack of beds and for the difficulty in managing critical and clinically unstable patients affected by a disease with phenotypic characteristics new to the whole scientific community. ${ }^{2}$ As a result, a very dangerous cap has been created in the ER, which was acutely overcrowded with no way out. This presence of many patients in the ER, in close contact with each other, has been a
\end{abstract}

Correspondence: Andrea Bellone, Department of Emergency Medicine, ASST-Grande Ospedale Metropolitano Niguarda, P.zza Ospedale Maggiore 3, 20161 Milano, Italy

Tel.: +390264447433

E-mail: andrea.bellone@ospedaleniguarda.it

Key words: Covid-19; Emergency; Overcrowding.

Acknowledgments: I thank you Jury Guaiana for supervising English.

Conflict of interest: the authors declare no potential conflict of interests.

Received for publication: 13 July 2020.

Accepted for publication: 16 November 2020.

This work is licensed under a Creative Commons Attribution 4.0 License (by-nc 4.0).

${ }^{(} C$ Copyright: the Author(s), 2020

Licensee PAGEPress, Italy

Emergency Care Journal 2020; 16:9242

doi:10.4081/ecj.2020.9242 source of infections between patients and patients, health workers and patients, and health workers and health workers. In addition, it favored a spread of infections outside the hospital. iii) Patients brought in by ambulances, waiting to enter the examination rooms of the ER, remained on stretchers creating a queue of ambulances unable to unload patients. iv) The exceptional congestion of the ER together with word of mouth reduced the number of emergency calls to the help line (118) - whose ability to respond effectively was already affect by lack resources - leading to patients being taken care of at home, mostly thanks to oxygen bought from local pharmacies. Some of them lost their lives at home. v) As far as Oro-Tracheal Intubation (OTI) is concerned, restrictive choices have been made in emergency areas and especially in ERs. Those choices were absolutely reasonable, but were mainly dictated by lack of available resources.

I do not believe I have the skills to establish where responsibility lies, but an analytical account of facts is the only way to avoid the risk of being overwhelmed by similar health emergencies in the future and to create the conditions for a constructive rethinking of such a fragile system.

The first real lesson, as we already knew, is to preserve the ER, creating all the ideal conditions in the hospital to avoid chronic overcrowding. We have been saying this for years, every winter, we witness our ERs being overcrowded and the hypocritical and guilty indifference of our administrators. The hospital as a whole must be redesigned in order to adapt its accommodation capacity to the incoming flows. To clarify, if 200 patients a day arrive in the hot area of the ER, we must ensure that all relevant hospital wards are able to take them under their responsibility - there has been a predictable and constant number over the last 10 years, even during emergencies - avoiding ER boarding of 12-24-48-72 hours. The relationship between elective and emergency activities in hospitals need to be redesigned so as to avoid unnecessary and harmful conflicts. Both organization and planning should be based on the hospitalized patient's functional care path, regardless of the self-certified needs of specific individuals. Emergency help line (118) activity loads should be reconsidered in order to reduce pressure on large hub-and-spoke hospitals. Now, but it's always been the right thing to do, it is mandatory to preserve the ER from overcrowding, to avoid possible outbreaks. We need to rethink spaces and paths in a virtuous way.

The second chapter is about primary care. Its fragility became evident during the Covid-19 emergency. In those months, and even beforehand, the citizen/patient lacked a trusted local point of reference. For years, Italians have been going to the ER rather than the GP. That's in $95 \%$ of cases. The main reason being that preventive medicine and basic diagnostics are largely unavailable. In addition to that, team work is no longer mandatory for GPs. But it is impossible to face any emergency, Covid-19 aside, without consulting other colleagues, sharing updates, knowledge, experience and without hours of 24/7 medical practice open to citizens. Certainly, the Covid-19 emergency was a hospital emergency, but now, during phase 2 , the role of primary care should be key in monitoring patients at home, contact tracing, alerting the ATS (Health Protection Agency) of suspect cases, activating virtuous 
primary/secondary care paths, avoiding the often-unnecessary recourse to ERs. Unfortunately, this is not the case, especially in Lombardy (Figure 1). The third aspect concerns the relationship between the NHS and private healthcare. In my opinion, this is not an ideological issue, but it's rather about putting medicine for the protection of the citizen's health first. For years, the corporatization of hospital facilities has distorted the concept of patient/person into a concept of patient/client. Budgets, cost reporting, regional reimbursements, competitions, and corporate strategies have bureaucratized hospitals and impoverished the most expensive structures (Emergency Departments), leading to a gradual deterioration, both in quantitative and qualitative terms, of emergency healthcare. As described above, during the Covid-19 epidemic, this led to its total collapse, after years of reprehensible lack of attention. In Lombardy, in particular, private healthcare accounts for about $50 \%$ of the entire regional health care system. Private healthcare can partly choose the services to be provided, regardless of the real demand of the citizen-patient, in order to make a greater profit through services better reimbursed by the regional health system. On the contrary, the bulk of emergency healthcare, in the red par excellence, has burdened the public system. This was also the case during the Covid-19 pandemic.

In conclusion, we have been paying for old bad habits inherited from years of dysfunctional health care: Before the Covid-19 emergency, little had been invested in ICUs, SICUs and Short Stay Observation (SSO), we had almost halved intensive units, and we had not invested in public health as much as other European countries, in relation to our GDP. In this dramatic emergency, the strate-

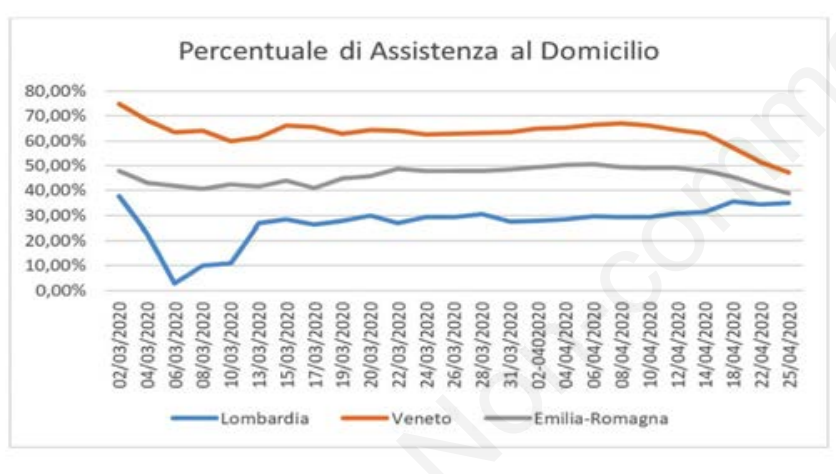

Figure 1. Percentage of home care in three different Italian regions. (Source: Maurizio Marangolo). gic and decisive role of ERs were highlighted as never before. Their endurance is a key factor in saving hospitals. We must: i) Preserve the ER as a place where professionals can be trained for excelling in their complex job. ii) Protect the ER from overcrowding and boarding (patient waiting in the ER due to lack of beds in the wards). iii) Incentivize first aid workers who work in a very delicate environment and whose activities, logistics and organization affect intra-hospital mortality too. iv) Let hospitals become containers where care paths are synchronized according to their ability to adapt to patients' needs (and not the other way around). v) Redesign the hospital and the ER so that they can guarantee safe logistics and routes for patients and health workers alike. vi) Make sure that primary and secondary care services are organically intevii) Create clinics with GPs working effectively on prevention, monitoring and basic diagnostics.

But the real challenge is to be prepared for any new pandemics. Not only do we need to think of hospitals with separate and spaced out paths, but we also need to think of multi-purpose areas with more specialists in dialogue with each other and different levels of care intensity. We really need to learn how to share skills, make use of different experience and get used to collective discussion. There is an urgent need for hospitals that are able to adapt quickly to new emergencies, including severe epidemics and/or situations particularly stressful for the health system. Cultural and organizational flexibility is the condition for rapid transformations and the necessary prerequisite to be prepared when necessary. My idea is to create filter zones before entering the hospital, in order to protect the ER from overcrowding and reduce the risk of turning the hospital into a source of infections. In addition to this, it is necessary to create two different paths, have rapid and reliable tests available, and create a medical/specialist area, depending on the intensity of care. It's also critical to make beds for patients coming out of ICUs, and for those who didn't need intensive care, immediately available and to imagine the creation of filter areas within the wards with single and/or negative pressure rooms. In this way, the hospital can become a safe space for the patient, from the entrance to the exit.

\section{References}

1. Rosembaum L. Facing Covid-19 in Italy-Ethics, logistics, and therapeutics on the epidemic's front line. New Engl J Med 2020; 382:1873-5

2. Gattinoni L, Chiumello D, Caironi P, et al. Covid-19 pneumonia: different respiratory treatments for different phenotypes? Intensive Care Med 2020:6-9. doi:10.1007/s00134-02006033-2 